\title{
Long-term effects of cutting versus non-cutting instruments in FESS*
}

\author{
L. Mus', R. Hermans², M. Jorissen' \\ Department of Otorhinolaryngology, University Hospitals Leuven, Leuven, Belgium \\ Department of Radiology, University Hospitals Leuven, Leuven, Belgium
}

Rhinology 50: 56-66, 2012

DOI: $10.4193 /$ Rhino 11.179

*Received for publication:

August 19, 2011

accepted: November 11, 2011

\section{Summary}

Background: Currently most ENT surgeons advocate using cutting instruments in Functional Endoscopic Sinus Surgery (FESS), based on the assumption of superior wound healing due to mucosal preservation. However, there is no scientific evidence of superiority of the cutting instruments.

Methods: A prospective, randomized double blinded study was performed in 100 patients undergoing FESS, operated one side with non-cutting and the contra lateral side with cutting instruments. After a mean follow-up of 12 years after FESS, unilateral and global sino-nasal symptoms were scored by the patients using VAS, endoscopic abnormalities scored at both sides at the Outpatient clinic and radiologic abnormalities were described.

Results: No significant differences between the sides operated by cutting and non-cutting instruments were found for sino-nasal symptomatic or for radiologic abnormalities between both sides. For the endoscopic parameters there was no difference for secretion or mucosa. Only for the presence of endoscopic synechiae there was a statistically significant difference between both groups. At the non-cutting side more synechiae were noticed, but no interference with sinus access, neither ventilation nor drainage was observed.

Conclusion: FESS performed with cutting instruments results in less synechiae formation compared to non-cutting instruments. However, the presence of synechiae is not associated with more symptoms or CT scan abnormalities.

Key words: paranasal sinuses, surgical procedures, surgical instruments, long-term effects

\section{Introduction}

Diseases of the nose and paranasal sinuses account for a large percentage of the cases seen by otorhinolaryngologists in clinical practice. Nowadays Functional Endoscopic Sinus Surgery (FESS) is the gold standard surgical treatment for inflammatory paranasal sinus pathology ${ }^{(1)}$. Surgery is tailored to the disease extent and concentrated on restoring mucociliary drainage and ventilation by opening the osteo-meatal complex prechambers of the major, dependent sinuses. Minimizing tissue trauma and preserving normal mucosa are paramount in avoiding excessive scarring or synechiae formation, which on its turn is an important determining factor of better healing and better outcome in the long-term ${ }^{(2)}$. Although Jankowski et al., promoted the opposite of the functional of FESS with nasalisation (radical ethmoidectomy). They suggest that in the treatment of nasal polyposis these more aggressive technique leads to better long-term results than FESS ${ }^{(3,4)}$.

The instruments used are considered to be an important determining factor in mucosal preservation. A theoretical advantage is hypothesized for the cutting instruments, although there are no examples of superiority found in evidence-based medicine. Moriyama et al.., ${ }^{(5)}$ first brought up the idea of superiority of the cutting instruments in mucosal preservation. In 
theory, cutting instruments are believed to remove diseased tissue more selectively leaving healthy mucosa behind lining the resulting cavities. Less denudation of bone would cause faster and improved healing together with better prevention of scar formation. On the contrary, non-cutting instruments are thought to be not delicate enough. They tend to grab and strip mucosa not selectively and consequently harm vital and functional mucosa. This leads to retained bone surfaces without mucosa, which can be the source of infection ${ }^{(1,5)}$. At present, ENT surgeons advocate using cutting instruments in FESS without scientific evidence of superiority.

Like any other procedure, it is coming under close scrutiny with relation to evidence-based efficiency. There is a great need to assess the efficiency of this procedure especially on a long-term basis. We wanted to test the validity of this hitherto unproven hypothesis. If the type of instrument plays a crucial role in the mucosal preservation and the cutting instruments are indeed more mucosa-saving, than we expect that on the long-term the subjective and objective evaluation of the nose and paranasal sinuses would have to differ.

In the period between November 1997 and August 1998, hundred selected patients with symmetric bilateral sinus disease underwent FESS. During surgery, cutting instruments have been used randomly on one side and non-cutting instruments on the contra lateral side. Previous studies reported short-term results (3 weeks after endoscopic surgery) ${ }^{(6)}$ and results 1 year after endoscopic surgery ${ }^{(7)}$. Neither of these two studies revealed a significant difference between the cutting side and the non-cutting side; neither for symptomatic nor for endoscopic evaluation. Although, the previously reported results have been plagued by two major shortcomings: relatively short follow-up and absence of systematic computed tomographic (CT) evaluation.

To date, a limited number of studies has reported on the postoperative mucosal healing after FESS by using CT scan for evaluation ${ }^{(8,9)}$. Although in literature much has been reported on the short-term outcomes of FESS, little has been reported with regard to its long-term impact on chronic sinusitis (4 years ${ }^{(10)}$, 5 years ${ }^{(11)}, 6$ years ${ }^{(12)}, 6.5$ years ${ }^{(13)}, 7.8$ years ${ }^{(14)}, 3-9$ years ${ }^{(15)}, 18$ years $\left.{ }^{(16)}\right)$. As some processes (e.g. synechiae, osteoneogenesis and mucocoeles) only become apparent after many years and some only can be described objectively on a CT scan, evaluation on the long-term including CT scan, might provide us with new insights.

The aim of this study was to perform detailed evaluation of sino-nasal symptoms, to evaluate endoscopic abnormalities, to examine processes only seen on $\mathrm{CT}$ and to analyse if there is a significant difference between the cutting and non-cutting side regarding better outcome in the long-term. Additionally we wanted to find out if a correlation exists between symptom severity, endoscopic score and CT scan score.

\section{Materials and methods}

\section{Patients}

The original cohort was composed of one hundred (100) patients, from one Belgian University Centre, with a diagnosis of bilateral symmetrical inflammatory sinus disease. There were 54 women and 46 men with a mean age of 44 years (range 14 - 77 years) at the time of surgery. Twenty-five patients had undergone previous sinus surgery, including a Caldwell-Luc operation, ESS procedures or multiple polypectomies. Indications for surgery, exclusion criteria and presence of associated diseases are displayed in Table 1.

\section{Operation procedure}

The operative procedures were performed under local or general anaesthesia from November 7, 1997 to August 5, 1998. All procedures were performed by the same surgeon (MJ). On the one side, there was a choice between the different cutting forceps (Blakesley, Grünwald-Henke), while on the other side there was just one non-cutting forceps (Weil-Blakesley). Uncinectomy was done using a sickle knife. Since there is no non-cutting backbiter, a cutting backbiter was always used when enlargement anteriorly of the antrostomy was needed. The anterior ethmoid was always addressed first and based on preoperative computer tomographic and endoscopic evaluations, additional regions were opened and cleared. There was random assignment cutting instruments were used for one side, while for the contra lateral side non-cutting instruments were used. Immediate postoperative treatment consisted of oral steroids, nasal douches and weekly endoscopic control with gentle suction cleanings. For details on the procedure we refer to previous publications ${ }^{(6)}$.

\section{Long-term follow-up procedure}

After approval of the ethical committee, follow-up visits were arranged from March 3, 2010 until September 28, 2010. The mean time interval between operation date and long-term follow-up visit was 12 years and 3 months (ranging from 11 y 6 $\mathrm{m}$ to 13 y $9 \mathrm{~m}$ ).

Fourteen patients dropped out (four patients died, ten patients could not be located). For all remaining patients ( $n=86$; mean age of 55 year; 47 women, 39 men) after they signed an informed consent, a questionnaire has been completed evaluating subjective symptoms. Twenty-seven of the patients were questioned by telephone, as they were not able or not interested to have an appointment at our centre. The other fifty-nine were questioned at the consultation. We asked if the patient underwent revision surgical procedures and how many times. For each surgery detailed information was collected about 
indication (mucocoele, localized or systemic pathology, ...), side (unilateral or bilateral) and the institution where it took place. We also asked the appreciation of the patient for nonlateralised (post nasal drip, sneezing, coughing and olfactory disturbance) and for lateralised symptoms (headache, maxillary pressure, secretions and nasal obstruction). The lateralised parameters were evaluated for each side on frequency $(0=$ never, 1 = occasional, 2 = continuous $)$ and severity ( 1 = little, 2 $=$ moderate, $3=$ severe). Post hoc a score for each parameter is analysed and it represents the product of frequency and severity (score 0 - 6 for each side). Additionally for each side a total symptom score is analysed, which represents the sum of the four parameters (score 0 - 24). The patients gave a global symptom score $(0=$ perfect, $1=$ very good, $2=$ moderate, 3 $=$ many complaints) to each side and indicated whether they experienced a difference between the sides.

In addition, at the consultation an experienced rhinologist, blinded of the side where cutting instruments had been used, performed a complete endoscopic evaluation, except in one patient who refused $(n=58)$. Three parameters (secretions, mucosa and synechiae) were checked in order to score the two sides. The score of the mucosa is the sum of four parameters (oedema, polyposis, inflammation and fibrosis, score 0 - 12). Grading the presence of each parameter between 0 and 3 (for secretions: absent, clear, mucoid or mucopurulent; for mucosa: absent, mild, moderate or severe; for synechiae: absent, single, multiple without obstruction or multiple with obstruction). Additionally for each side a total endoscopic score is analysed, which represents the sum of the three parameters (score 0 - 18). The rhinologist gave a global endoscopic score $(0=$ perfect, 1 = very good, 2 = moderate, $3=$ severe) to each side and indicated whether he noted a difference between the sides.

Subsequently, a CT scan was performed by fifty-seven patients (two pregnant women were excluded). The scans were reviewed by a radiologist, who was blinded to side difference of cutting and non-cutting instruments and to the patients' clinical characteristics, he recorded four anatomical abnormalities. First the extent of soft tissue densities was scored according to the Lund-Mackay scoring system $(0-12)^{(17)}$. Second a lateralization of the middle or superior turbinate graded was described with a three point score $(0=$ no lateralization, $1=$ limited without obstruction, 2 = extensive lateralization with obstruction), because this may reflect scar formation ${ }^{(18)}$. When a turbinate is absent a score of 0 is given. The third parameter is the presence of osteoneogenesis at each sinus group (maxillary, anterior and posterior ethmoid, sphenoid and frontal). This is scored with a four-point score system ( $0=$ no, 1 = minimal, 2 = extensive, 3 = complete area). Finally, we looked for mucocoeles (defined as a completely opacified sinus or cell with evidence of expansion). For each sinus group the absence
$(=0)$ or presence $(=1)$ is indicated. In case of revision surgery, the CT scan prior to revision was also analysed and taken into account for osteneogenesis, mucocoele and lateralisation of turbinates. Additionally, when a mucocoele or osteoneogenesis was found, a comparison with the CT scan before surgery at 1997-1998 was done. Additionally, for each side a total radiographic score is analysed, which represents the sum of the four parameters (score 0 - 39). The radiologist gave a global radiographic score $(0=$ perfect, $1=$ very good, $2=$ moderate, $3=$ severe) to each side and indicated whether he noted a difference between the sides.

\section{Statistics}

Statistical analysis was done using SPSS software and Excel. The cutting and non-cutting side were compared with the Wilcoxon signed-rank test (2 paired samples), two sided at the $5 \%$ level of significance. The null hypothesis for each observed parameter is that there is no difference between the scores of the cutting side and the scores of the non-cutting side. Each patient served as his own control, eliminating all possible confounding effects based on differences between the subjects (degree of disease, associated diseases).

Additionally, Spearman correlation coefficients were used to evaluate the correlation between global symptom severity, global endoscopic score and global CT scan score. We determined the $p$ values, also with a $p<0.05$ set to achieve statistical significance.

\section{Results}

Below we describe the results of lateralised parameters (respectively sino-nasal symptoms, endoscopic and radiologic abnormalities). Each time we made an analysis of lateralised symptoms/signs, a total score (sum of the evaluated parameters) and a global score (subjective overall experience score 0 - 3). Additionally, we give results of revision surgery and we describe correlation between symptomatic, endoscopic and radiographic analysis.

For an overview of the $p$-values and the mean values of the analysed parameters we refer to Tables 2 and 3.

\section{A. Lateralised symptoms ( $n=86$ patients)}

Individual analysis of four lateralised symptoms was performed (score 0 - 3 for each side) (Figure 1). There was no statistically significant difference between the cutting and non-cutting side for each parameter (headache $p=0.752$; maxillary pressure $p=0.928$; secretions 0.959 ; nasal obstruction $p=0.495$ ). On the sum of these four parameters (total symptom score: 0 - 24 for each side) (Figure 2). could no statistically significant difference be detected $(p=0.532)$. Neither the global symptom score (score 0 - 3) to each side could give a significant difference $(p=0.973)$.

A separate analysis of the patients questioned only by te- 
Table 1. Demographic data.

\begin{tabular}{|c|c|}
\hline \multicolumn{2}{|l|}{ Distribution of indication for surgery } \\
\hline Chronic rhinosinusitis without polyps & $43 \%$ \\
\hline Chronic rhinosinusitis with polyps & $35 \%$ \\
\hline Recurrent acute rhinosinusitis & $18 \%$ \\
\hline Miscellaneous indications & $4 \%$ \\
\hline \multicolumn{2}{|l|}{ Exclusion criteria } \\
\hline \multicolumn{2}{|l|}{ Bilateral asymmetric disease } \\
\hline \multicolumn{2}{|l|}{ Paranasal sinus tumorus } \\
\hline \multicolumn{2}{|l|}{ Paediatric patients ( $<12$ years) } \\
\hline \multicolumn{2}{|l|}{ Cystic fibrosis or ciliary dysfunction } \\
\hline \multicolumn{2}{|l|}{ Immune-compromised patiens } \\
\hline \multicolumn{2}{|l|}{ Presence of associated diseases } \\
\hline Asthma & $22 \%$ \\
\hline Allergy & $15 \%$ \\
\hline APA syndrome & $1 \%$ \\
\hline
\end{tabular}

Indications for surgery, exclusion criteria and presence of associated diseases.

lephone $(n=27)$, neither indicated a statistically significant difference (headache $p=1$; maxillary pressure $p=1$; secretions $p=0.157$; nasal obstruction $p=0.197$; total score $p=1$; global score $p=0.256)$.

\section{B. Endoscopic findings ( $n=58$ patients)}

Individual analysis of three endoscopic parameters was performed (Figure 3) and no sinus specific changes were found. There was no statistically significant difference between the side operated by cutting and non-cutting side for secretions $(p=0.655)$ or mucosa $(p=0.632)$. A statistically significant difference was found for the synechiae $(p=0.005)$.

Further we calculated a total endoscopic score (sum of the three endoscopic parameters, score 0 - 18 for each side) (Figure 4) and made a global symptom score ( 0 - 3). We could not detect a statistically significant difference (respectively $p=$ 0.063 and $p=0.083$ ).

Because of the significant difference, we further investigated on the synechiae. Fifteen out of the fifty-eight patients had synechiae (26\%) and none of them had undergone revision surgery. For the extent of the formed synechiae we made a difference between single synechia without obstruction $(n=13)$, multiple synechiae without obstruction $(n=2)$ and multiple with obstruction $(n=0)$. We also specified the localization of the synechiae. Three patients had an equal score for synechiae at the cutting and the non-cutting side and the synechiae were located on the same spot (between the middle turbinate and the lateral nasal wall $(n=2)$ or within the ethmoid sinus cavity $(n=1))$. Only one patient had a synechia at the cutting side (while none were noted at the non-cutting side), which was localized between the septum and the inferior turbinate.

One patient had symmetric synechiae between the middle turbinate and the lateral nasal wall and an extra synechia at the non-cutting side in the maxillary sinus access. The other ten patients had at least one synechia at the non-cutting side (while none at the cutting-side). One synechia was found within the ethmoid sinus cavity and all other synechiae were found between the middle turbinate and the lateral nasal wall. Further exploration of the data for these eleven patients revealed no consequently higher score on the symptomatic, endoscopic or radiologic parameters than the contra lateral side. Based on this, we conclude there are no clinical consequences of the synechiae.

Furthermore, we compared the current endoscopic results on synechiae with those from 1999 (one year after surgery). In 1999 , synechiae were endoscopically evaluated in 22 patients out of $88(25 \%)$. Six of them were dropped out in the follow-up study. Two out of twenty had same synechiae now and twelve years ago, none of these underwent revision surgery. One patient had more synechiae; he had no revision surgery. But at least eleven patients had no synechiae anymore. Remark that only one out of these eleven patients underwent revision surgery at his non-cutting side, the indication was multiple synechiae. Another patient underwent revision surgery at his cutting side, but synechiae were present at the non-cutting side. The current study revealed synechiae for an extra 12 patients (one patient was not evaluated in 1999).

\section{CT findings ( $\mathrm{n}=57$ patients)}

The occurrence of five radiographic abnormalities was individually studied (Figure 5). No significant difference comparing the two surgical instruments was found (opacification $p=$ 0.657 , lateralisation of the middle or superior turbinate $p=1$, mucocoeles $p=1$, osteoneogenesis $p=1$ ). Two patients with osteoneogenesis were excluded, because none was related to the use of the instruments during surgery. One patient had a complete osteoneogenesis of the left frontal sinus. Remark that this could not be caused by the used instruments as the surgeon did not enter into the frontal sinus. The second patient had bilateral maxillar osteoneogenesis due to surgery before 1998 (bilateral Cald-well Luc procedure).

Again, no statistically significant difference could be detected on the total score $(0-39)$ (Figure 6$)$ nor on the global radiographic score $(0-3)(p=0.657$ and $p=0.366$, respectively) to each side. 
Table 2. P-values ( $p<0.05$ set to achieve statistical significance).

$P$-values for total score

\begin{tabular}{|c|c|c|c|}
\hline & Symptomatic & Endoscopic & CT scan \\
\hline Total & $P=0.532$ & $P=0.063$ & $P=0.657$ \\
\hline Telephone $(n=27)$ & $P=0.256$ & l & I \\
\hline Revision surgery $(n=11)$ & $P=0.785$ & $P=0.197$ & $P=0.589$ \\
\hline
\end{tabular}

P-values for global score (subjective overall experience, score 0 - 3)

\begin{tabular}{|c|c|c|c|}
\hline & Symptomatic & Endoscopic & CT scan \\
\hline Total & $P=0.973$ & $P=0.083$ & $P=0.366$ \\
\hline Telephone $(n=27)$ & $P=1.000$ & / & / \\
\hline Revision surgery $(n=11)$ & $P=0.317$ & $P=0.157$ & $P=1.000$ \\
\hline
\end{tabular}

P-values for parametric-specific symptomatic analysis

\begin{tabular}{|lcccc|}
\hline & Headache & Maxillar pressure & Secretions & Nasal obstruction \\
\hline Total $(n=86)$ & $P=0.752$ & $P=0.928$ & $P=0.959$ & $P=0.495$ \\
Telephone $(n=27)$ & $P=1.000$ & $P=1.000$ & $P=0.157$ & $P=0.197$ \\
\hline Revision surgery $(n=11)$ & $P=0.655$ & $P=1.000$ & $P=0.317$ & $P=0.655$ \\
\hline
\end{tabular}

P-values for parametric-specific endoscopic analysis

\begin{tabular}{|c|c|c|c|}
\hline & Secretions & Mucosa & Synechiae \\
\hline Total $(n=58)$ & $P=0.655$ & $P=0.632$ & $P=0.005$ \\
\hline Revision surgery $(n=9)$ & $P=0.317$ & $P=0.197$ & $P=1.000$ \\
\hline
\end{tabular}

P-values for parametric-specific radiographic analysis

\begin{tabular}{|c|c|c|c|c|}
\hline & Opacification & $\begin{array}{l}\text { Lateralisation } \\
\text { turbinates }\end{array}$ & Mucoceles & Osteoneogenesis \\
\hline Total $(n=57)$ & $P=0.657$ & $P=1.000$ & $P=1.000$ & $P=1.000$ \\
\hline Revision surgery $(n=9)$ & $P=0.589$ & $P=1.000$ & $P=1.000$ & $P=1.000$ \\
\hline
\end{tabular}

To exclude bias we calculated $p$-values respectively for the total population, the patients questioned only by telephone and the patients who underwent revision surgery.

Total score is the sum of the lateralised parameters, respectively anamnesis (headache, maxillar pressure, secretions and nasal obstructions), endoscopy (secretions, mucosa, synechiae) and CT scan (opacification, la teralisation of turbinates, presence of mucoceles and osteoneogenesis). 


\begin{tabular}{|c|c|c|c|}
\hline & Mean score & Mean score & P-value \\
\hline ANAMNESTIC $(n=86)$ & Cutting side & Non-cutting side & \\
\hline Headache (score 0 - 6) & 0.90 & 0.87 & NS \\
\hline Maxillary pressure (score 0 - 6 ) & 0.71 & 0.66 & NS \\
\hline Secretions (score 0 - 6) & 1.47 & 1.42 & NS \\
\hline Nasal obstruction (score 0 - 6) & 1.21 & 1.30 & NS \\
\hline Total symptomatic score (score 0 - 24) & 4.28 & 4.26 & NS \\
\hline Global symptomatic score (score 0 - 3) & 1.35 & 1.34 & NS \\
\hline
\end{tabular}

\begin{tabular}{lccc} 
ENDOSCOPIC $(\mathbf{n}=\mathbf{5 8})$ & \multicolumn{2}{c}{ Cutting side } & \multicolumn{2}{c}{ Non-cutting side } \\
\hline Secretions (score 0-3) & 0,36 & 0,38 & NS \\
Mucosa (score 0-12) & 0,83 & 0,90 \\
Synechiae (score 0-3) & 0,09 & 0,28 \\
Total endoscopic score (score 0-18) & 1,28 & 1,55 \\
Global endoscopic score (score 0-3) & 0,55 & 0,66 & NS
\end{tabular}

\begin{tabular}{lcc} 
CT SCAN $(\mathbf{n}=\mathbf{5 7})$ & Cutting side & Non-cutting side \\
\hline Opacification (score 0 - 12) & 1.60 & 1.72 \\
Lateralisation of middle turbinate (score 0 - 2) & 0.00 & 0.00 \\
Lateralisation of superior turbinate (score 0 - 2) & 0.00 & 0.00 \\
Mucocoele (score 0 - 4) & 0.00 & 0.00 \\
Osteoneogenesis (score 0 - 15) & 0.00 & 0.00 \\
Total radiographic score (score 0 - 39) & 1.60 & NS \\
Global radiographic score (score 0 - 3) & 0.53 & NS \\
\hline
\end{tabular}

These tables represent the mean scores for each parameter, respectively for the side where cutting instruments were used and for the side where non-cutting instruments were used. We indicated the p-value as no significant (NS) and significant $\left(^{*}\right)$.

\section{Other variables}

Eleven out of the eighty-six patients we questioned had surgical failure and needed at least one revision surgery (nine patients one revision surgery, one patient two revision surgeries, one patient three revision surgeries). Two patients reported revision surgery at their cutting side, four patients at their non-cutting side and five patients on both sides. We note that two out of eleven could only be evaluated by telephone, the other nine were further evaluated with endoscopy and CT scan. Separate analysis of these nine patients did not confirm a statistically significant difference for parametric-specific scores nor for total or global scores (Table 2).

Additionally, we tried to find a correlation between symptomatic, endoscopic en radiographic analyses. Figure 7 gives an overview of the global scores, split up between cutting and non-cutting side. After a statistical analysis to find a correlation between these global scores, a very weak correlation was confirmed between global symptomatic score and global endoscopic score (Spearman correlation coefficient cutting side $0.337(p=0.01)$ and non-cutting side $0.261(p=0.048)$ ). Statistical analysis confirmed no correlation between global symptomatic score and global radiographic score (Spearman correlation coefficient cutting side $0.120(p=0.373)$ and non-cutting side $0.063(p=0.639)$ ). Finally, statistical analysis confirmed moderate correlation between global endoscopic score and global radiographic score (Spearman correlation coefficient cutting side $0.464(p<0.01)$ and non-cutting side $0.543(p<0.01))$.

\section{Discussion}

In the present study, we evaluated the effects of instrument type (cutting versus non-cutting) used during on the longterm outcome. The results suggest that overall on the longterm no difference between the use of cutting and non-cutting instruments is noted. No significant difference could be calcu- 


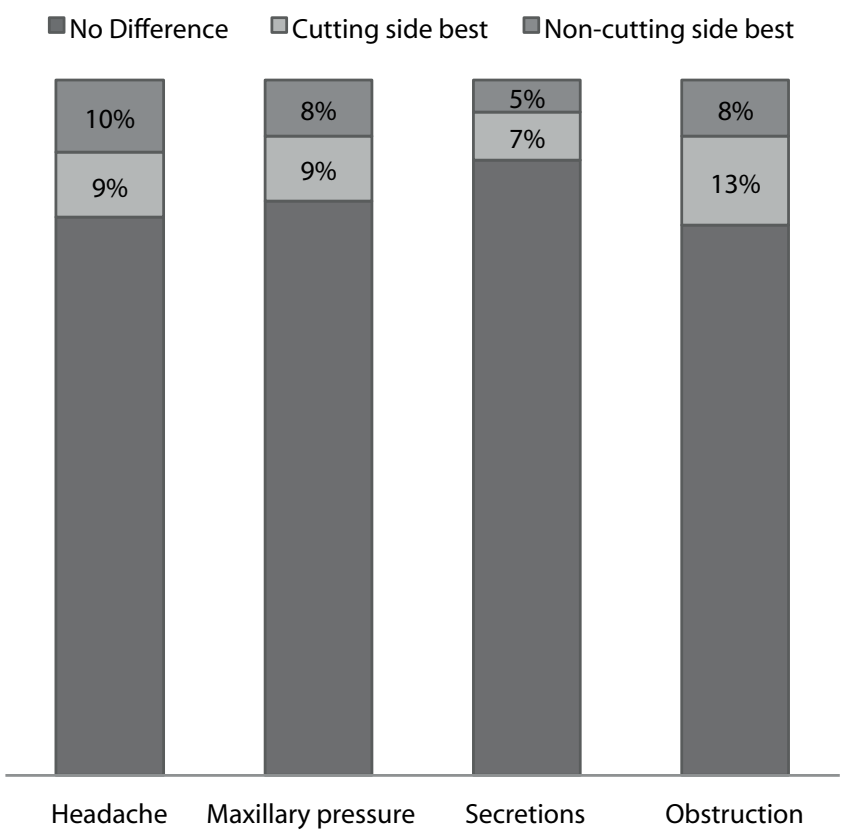

Figure 1. Showing for each lateralised symptom the percentage of patients that indicated respectively no difference between the side where cutting instruments were used and the side where non-cutting instruments were used, patients who indicated the cutting side as better and patients who indicated non-cutting side as better.

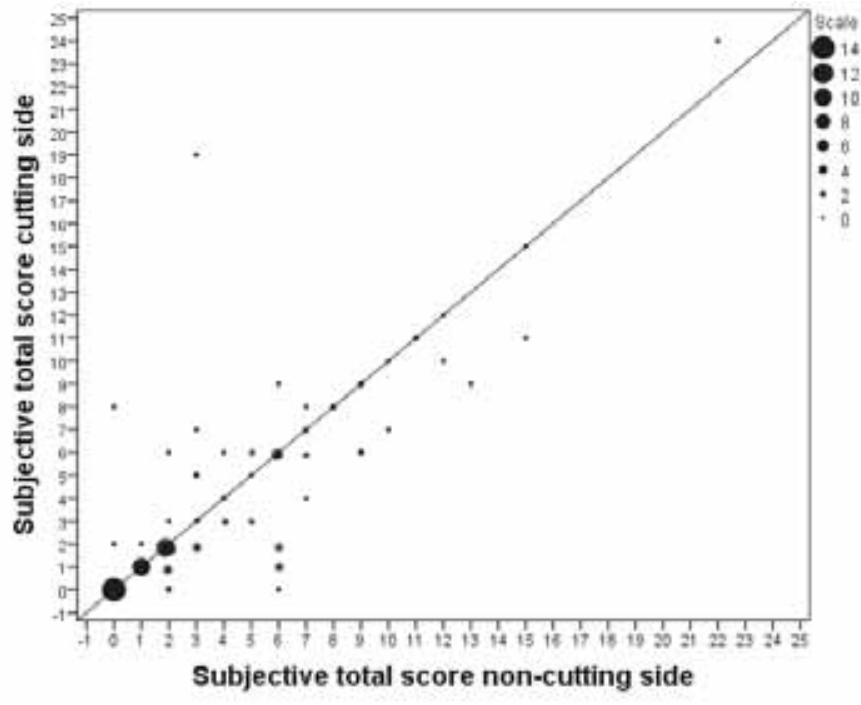

Figure 2. Showing the distribution of the total symptom scores (score 0 - 24). The $X$-axis represents the total score for the non-cutting side, the Y-axis represents the total score for the cutting side. The scale of each point indicates for the amount.

$\square$ No Difference $\quad \square$ Cutting side best $\quad \square$ Non-cutting side best

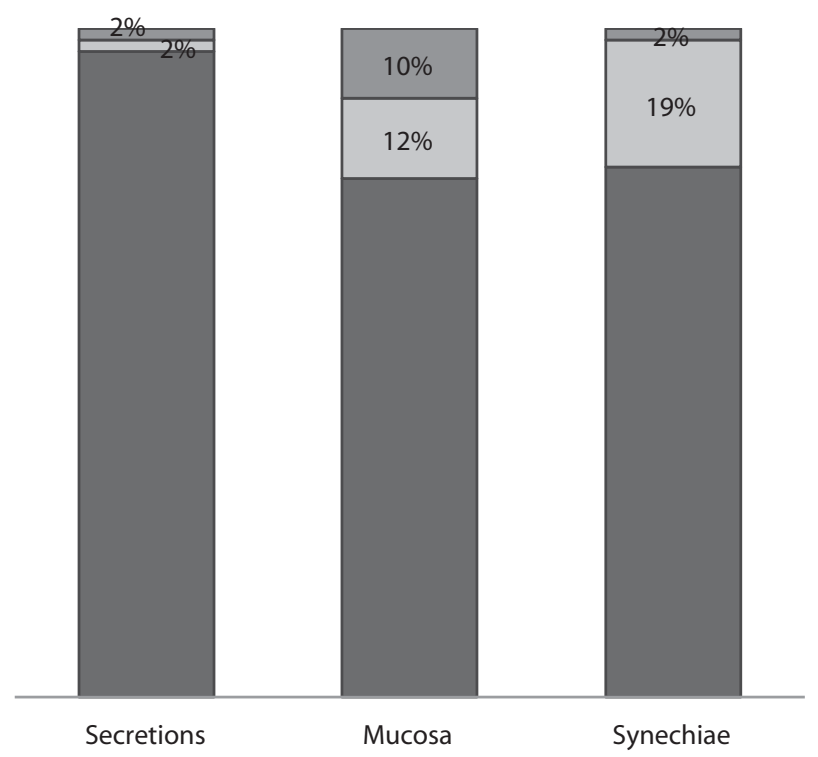

Figure 3. Showing for each lateralised endoscopic parameter the percentage of patients by whom respectively no difference was indicated by the rhinologist between the side where cutting instruments were used and the side where non-cutting instruments were used, patients by whom the cutting side was indicated as better and patients by whom the non-cutting side was indicated as better.

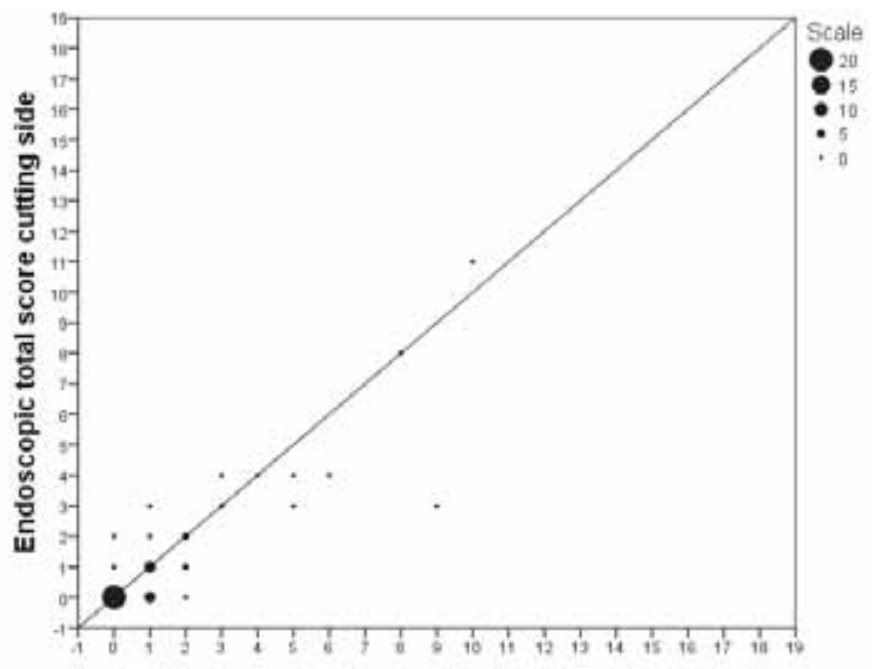

Endoscopic total score non-cutting side

Figure 4. Showing the distribution of the total endoscopic scores score 0 - 18). The $X$-axis represents the total score for the non-cutting side, the Y-axis represents the total score for the cutting side. The scale of each point indicates for the amount. 
lated for total score (representing the sum of respectively all lateralised symptoms, endoscopic and radiographic findings) nor for global score (subjective overall experience, score 0 - 3). A separated analysis of each lateralised symptom (headache, maxillary pressure, secretions and nasal obstruction), endoscopic finding (secretions and mucosal alterations) and radiologic abnormalities (opacification, scar formation, osteoneogenesis and mucoceles) did not reveal either a statistically significant difference. Only the endoscopic presence of synechiae confirmed a statistically significant difference between the cutting and non-cutting side.

In addition, the previous studies on the postoperative symptomatic and endoscopic follow-up in the first three weeks and one year after surgery could not confirm the claimed theoretical advantage of the cutting instruments. Actually, those previous reports did not reveal the significant difference for synechiae.

Note that scarring and synechiae formation are the most common minor complications after FESS. Synechiae formation occurs when two opposing damaged mucosal surfaces are allowed to adhere during the healing process. They are often asymptomatic, but depending on the extent and localisation of the formed synechiae, symptomatic sinus outflow tract obstruction and recurrence of the disease may result ${ }^{(19-21)}$. Mucosal damage may have implications appear only on long term; so long term follow-up is required to evaluate synechiae. Further investigation of the synechiae found in our study confirmed that most were localised between the middle turbinate and the lateral nasal wall. Healing after sinus surgery is of utmost importance within the frontal recess area. Here we found no evidence of a sinus specific difference between the use of cutting and non-cutting instruments. Seen this particular localisation, we can conclude that this has no interference with sinus access, ventilation or drainage. This is confirmed by the observation that the side of synechiae was not consequently scored higher than the other side for the lateralized symptomatic, endoscopic and radiologic parameters. Even more, comparison of the current endoscopic synechiae data with the endoscopic data of one year after surgery revealed that synechiae evolve through time. Out of the fifteen patients in the current study noted with synechiae, only two were evaluated with equal synechiea, one with more synechiae and eleven with newly formed synechiae (note that one patient was not evaluated in 1999). None of these fifteen patients underwent revision surgery.

Additionally, we report on the correlations between subjective and objective findings. To date, there are only few studies that reported on the correlations between subjective and objective findings in the post-surgical patient population ${ }^{(8,9)}$. We noted similar results, i.e. on the one hand symptoms had poor correlation with endoscopic and CT scores and on the other hand objective clinical variables of endoscopy and sinus CT had moderate correlation. This suggests that endoscopy conveys similar information to CT in post-ESS patients. Endoscopy may not allow visualization of the full extent of disease as afforded by radiologic studies, but endoscopy likely yields more diagnostic information in the post-ESS patient given the greater access to the sinus cavities.

The following points regarding the current study need to be considered. Although the number of patients lost to followup from the originally reported cohort was small, one has to consider that this is not a large-scale study. Finally, only fifty-seven patients were completely examined (anamnesis, endoscopic and (T-scan). Rare or infrequent complications (such as osteoneogenesis) eventually may have been missed. But, we had anamnestic analysis of more than $85 \%$ of original cohort and endoscopic and CT scan evaluation of more than half of the original population. No bias was noted on the twenty-seven patients questioned only by telephone. Bearing these remarks in mind we can conclude the findings of these study are relevant.

Second, we remark the limitation in subjective analysis, because it takes only the lateralised symptoms into account. It is not possible to make conclusions about non-lateralised symptoms as postnasal drip, smell, coughing and sneezing. But considering the other findings, it looks unlikely that this would have led to different observations and conclusions.

Another problem is the fact that the outcome measures are open to individual interpretation and bias as there are no objective parameters for symptomatic and endoscopic evaluation. Nasal conditions are difficult to quantify as the symptoms only correlate poorly with objective signs. This could be the reason that symptoms do not correlate with objective signs of CT scan and that endoscopic scores just have moderate correlation with CT scan scores. The advantage of our study is that almost all patients were questioned by the same doctor (except five patients), endoscopic evaluation was done by the same rhinologist (except three patients) and CT scans were all evaluated by the same radiologist. An additional strength of our study lays in the used materials and methods as each patient served as his own control. So there is no possibility of confounding by other variables (e.g. pathology type, degree of disease, individual interpretation).

Another point to consider is that previous and revision surgery might bring a possible bias. Twenty-five patients underwent previous sinus surgery (Caldwell-Luc operation, ESS or multiple polypectomies) and eleven patients underwent revision 
$\square$ No Difference

$\square$ Cutting side best $\square$ Non-cutting side best

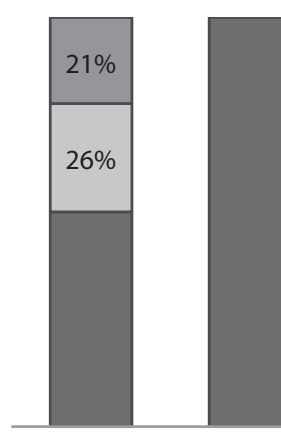

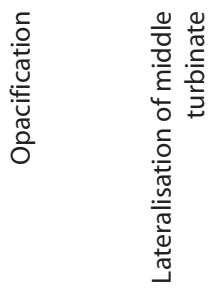

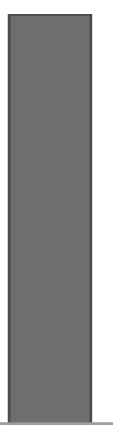

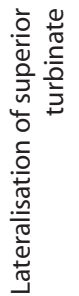

Figure 5. Showing for each lateralised radiographic parameter the percentage of patients by whom respectively no difference was indicated by the radiologist between the side where cutting instruments were used and the side where non-cutting instruments were used, patients by whom the cutting side was indicated as better and patients by whom the non-cutting side was indicated as better.

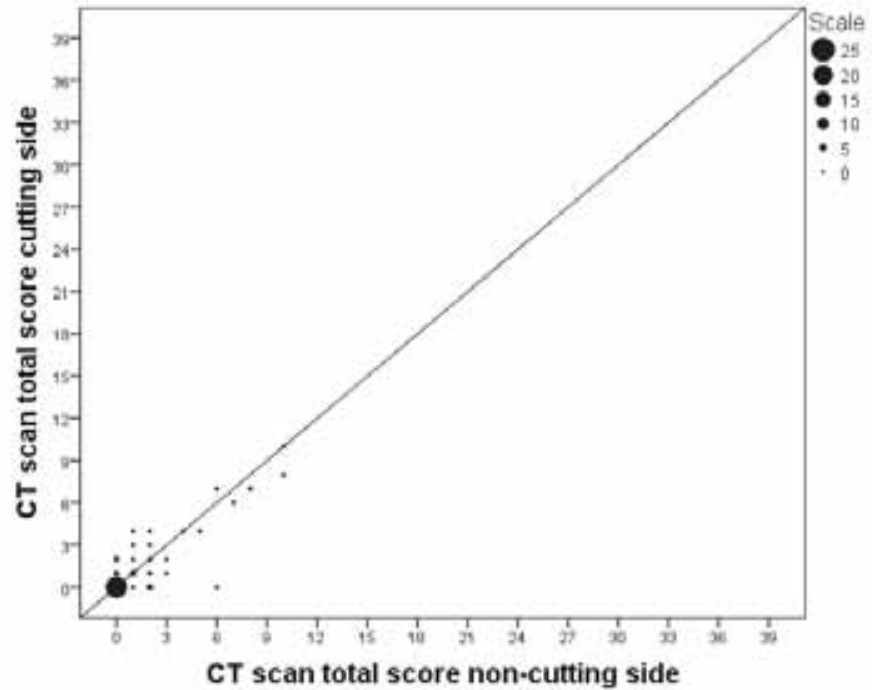

Figure 6. Showing the distribution of the total CT-scan scores (score 0 - 39). The $X$-axis represents the total score for the non-cutting side, the Y-axis represents the total score for the cutting side. The scale of each point indicates for the amount.

$\square$ Perfect Minor $\square$ Moderate $\square$ Severe

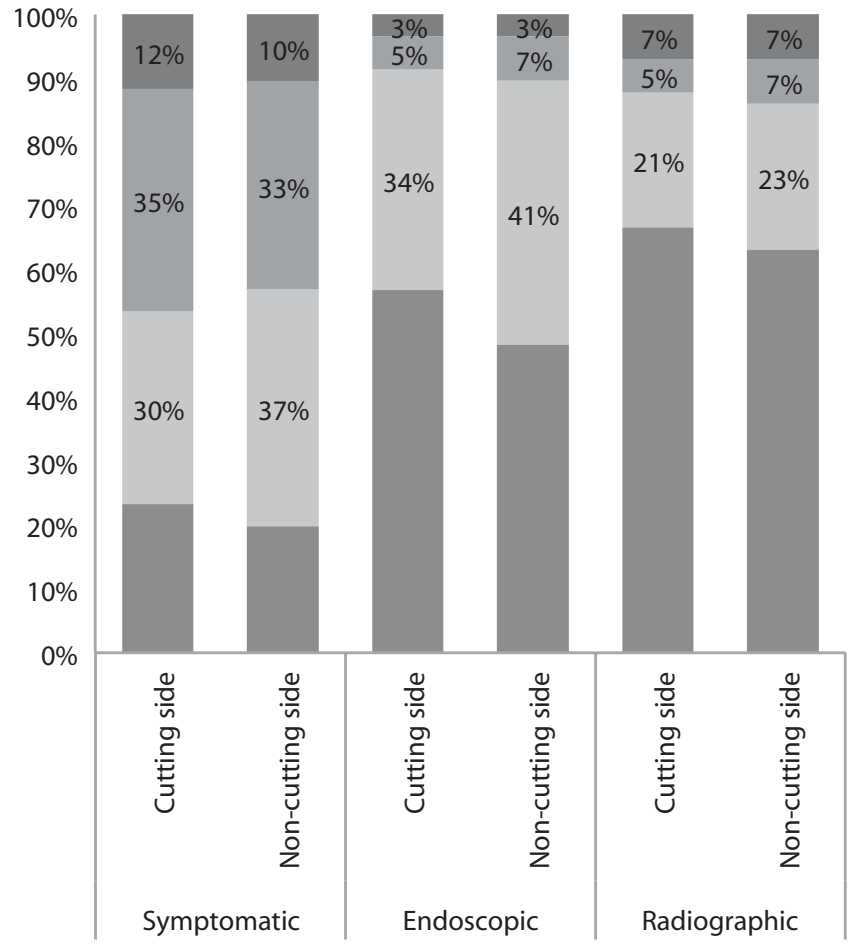

Figure 7. Showing an overview of the global scores (score $0=$ perfect, 1 = minor complaints, 2 = moderate complaints, 3 = severe complaints), calculated for symptomatic, endoscopic or radiographic evaluation and split between cutting and non-cutting side. 
surgery (revision FESS, DRAF III procedures, Denker method). However, the fifteen patients with synechia underwent no revision surgery, so no correlation could be found. Finally, we note that no similar studies comparing cutting with non-cutting instruments can be found in literature to support our findings. Today, alternatives for the standard non-cutting instruments are available, such as cutting instruments, powered instruments and surgical lasers ${ }^{(22)}$. Only a few similar studies have been published for other devices (i.e. powered instruments ${ }^{(23,24)}$ and holmium-YAG ${ }^{(25)}$ or KTP lasers $\left.{ }^{(26)}\right)$. Currently, none of those could offer statistical proven advantages over conventional instruments for better sinus surgery outcomes ${ }^{(22)}$. Although in literature, few results on long-term postoperative follow-up (10-16) are available, none of these reports are comparative nor similar to our study.

One must know that there are several factors that determine the outcome of the healing: pathology (chronic sinusitis vs nasal polyps), surgeons' technique and postoperative medical therapy. Specifically long-term use of local steroids is beneficial in case of nasal polyposis ${ }^{(27)}$.

Today, several authors consider the used instruments as a crucial element for the achievement of unlimited resection, while maximally preserving normal mucosa to create adequate ventilation combined with optimal mucociliary clearance. ENT surgeons advocate the cutting instruments for the mucosal preservation. However, this study suggests that the instruments were not the determining factor in long-term outcome after surgery. So one can do FESS according to the state of art using non-cutting instruments. But probably delicate tissue handling of the surgeon is more important than the actual tool used to perform the surgery.

\section{Conclusion}

We can conclude that FESS with non-cutting instruments has more synechiae formation on the long term compared to cutting-instruments. However, these synechiae have no interference with sinus access, ventilation or drainage. Additionally, the evaluation of symptoms and CT scan abnormalities on the long-term did not result in a statistically significant difference between the treated cutting and non-cutting side. So our study could not confirm the theoretical advantages of the cutting instruments. Based on the present observations one can do FESS according to the state of art using non-cutting instruments.

\section{Acknowledgement}

We would like to thank radiologist Dr. M. Koolen for his assistance in evaluating the CT scan (Department of Radiology, University Hospitals Leuven, Herestraat 49, 3000 Leuven, Belgium).

\section{Author contribution}

LM: study design, data collection, data analysis, and manuscript preparation (main author), $\mathrm{RH}$ : data collection (radiology), reviewer, MJ: assistance with study design, data collection (endoscopy), writing assistance.

\section{Conflict of interest}

No conflict of interest exist.

\section{References}

1. Kennedy DW, Senior BA. Endoscopic sinus surgery: a review. Otolaryngol Clin North Am. 1997; 30: 313-330.

2. Bernstein J, Lebowitz R, Jacobs J. Initial report on postoperative healing after endoscopic sinus surgery with the microdebrider. Otolaryngol Head Neck Surg. 1998; 118: 800-803

3. Jankowski R, Pigret D, Decroocq F Comparison of functional results after ethmoidectomie and nasalization for diffuse and severe nasal polyposis. Acta Otolaryngol (Stockh). 1997; 117: 601-608.

4. Jankowski R, Pigret D, Decroocq F, et al. Comparison of radical (nasalisation) and functional ethmoidectomy in patients with severe sinunasal polyposis. A retrospective study. Rev Laryngol Otol Rhinol (Bord). 2006; 127: 131-140.

5. Moriyama $\mathrm{H}$, Yanagi $\mathrm{K}$, Ohtori $\mathrm{N}$ et al. Healing process of sinus mucosa after endoscopic sinus surgery. Am J Rhinol. 1996; 10: 61-66.

6. Vauterin T, Vander Poorten V, Jorissen M Cutting forceps in functional endoscopic sinus surgery. Acta Otorhinolaryngol Belg. 2000; 54: 29-37.

7. Vauterin $T$, Vander Poorten $V$, Jorissen $M$. Long term effects of cutting forceps in endoscopic sinus surgery. Rhinology. 2006; 44: 123-127.

8. Bonfils P, Tavernier L, Abdel Rahman $H$, Mimoun M, Malinvaud D. Evaluation of combined medical and surgical treatment in nasal polyposis -III. Correlation between symptoms and CT scores before and after surgery for nasal polyposis. Acta OtoLaryngologica. 2008; 125: 318-323.

9. Ryan WR, Ramachandra T, Hwang PH. Correlations between symptoms, nasal endoscopy, and in-office computed tomography in post-surgical chronic rhinosinusitis patients. Laryngoscope. 2011; 121: 674-978.

10. Schaitkin B, May M Shapiro A, Fucci M, Mester SJ. Endoscopic Sinus surgery: 4-year follow-up on the first 100 patients. Laryngoscope. 1993; 103: 1117-1120.

11. Rowe-Jones JM, Medcalf M, Durham SR, Richards DH, Mackay IS. Functional endoscopic sinus surgery: 5 year follow up and results of prospective, randomized, stratified, double-blind, placebo controlled study of postoperative fluticasone propionate aqueous nasal spray. Rhinology. 2005; 43: 2-10.

12. Rowe-Jones JM, Mackay IS. Endoscopic Sinus surgery in the treatment of cystic fibrosis with nasal polyposis.Laryngoscope. 1996; 106(12 Pt 1): 1540-1544.

13. Senior BA, Kennedy DW, Tanabodee J, Kroger H, Hassab M, Lanza DC. Long-term impact of functional endoscopic sinus surgery on asthma. Otolaryngol Head Neck Surg. 1999; 121: 66-68.

14. Senior BA, Kennedy DW, Tanabodee J, et al. Long-term results of functional endoscopic sinus surgery. Laryngscope. 1998; 108: 151-157

15. Giger R, Dulguerov P, Quinodoz D, et al. Chronic panrhinosinusitus without nasal polyps: Long-term outcome after functional endoscopic sinus surgery. Otolaryngol Head Neck Surg. 2004; 131: 534-541.

16. Danielsen A, Olofsson J. Endoscopic endonasal sinus surgery: a review of 18 years of practice and long-term follow-up. Eur 
Arch Otorhinolaryngol. 2006; 263: $1087-$ 1098.

17. Lund VJ, Kennedy DW. Staging for rhinosinusitus. Otolaryngol Head Neck Surg. 1997; 117: S35-40.

18. Huang B, Lloyd K, DelGaudio J, Jablonowski E, Hudgins P. Failed Endoscopic Sinus Surgery: Spectrum of CT Findings in the Frontal Recess. RadioGraphics. 2009; 29: 177-195

19. Xiao J, Li Y, Zhang G. The correlative factors and preventive management of synechiae following endoscopic sinus surgery. Zhonghua Er Bi Yan Hou Ke Za Zhi. 1999; 34: 356-358.

20. Jae Yong Lee, MD, PhD, Seung Won Lee, MD. Preventing lateral synechia formation after endoscopic sinus surgery with a silastic sheet. Arch Otolaryngol Head Neck Surg. 2007; 133:776-779.

21. Bhalla RK, Kaushik V, de Carpentier Conchopexy suture to prevent middle turbinate lateralization and septal haematoma after endoscopic sinus surgery. Rhinology. 2005; 43: 143-145.

22. Fokkens W, Lund V, Mullol J, et al. European Position Paper on Rhinosinusitus and Nasal Polyps. 2007; suppl 20: 70-71.

23. Sauer $M$, Lemmens $W$, Vauterin $T$, Jorissen $M$. Similar surgical outcome in endoscopic sinus surgery by using the Microdebrider versus Classical instruments: a doubleblind randomised study. B-ENT. 2007; 3:1-7.

24. Selivanova O, Kuehnemund M, Mann WJ, Amedee RG. Comparison of conventional instruments and mechanical debriders for surgery of patients with chronic sinusitis. Am J Rhinol. 2003, 17: 197-202.

25. Metson R. Holmium.YAG laser endoscopic sinus surgery: a randomized, controlled study. Laryngoscope. 1996; 106 (1 Pt 2 suppl 77): 1-18.

26. Gerlinger I, Lujber L, Jarai T, Pytel J. KTP-532 laser-assisted endoscopic nasal sinus sur- gery. Clin Otolaryngol. 2003; 28: 67-71.

27. Jorissen $M$ and Bachert C. Effect of corticosteroids on wound healing after endoscopic sinus surgery. Rhinology. 2009; 47; 280-286.

\section{Lisabeth Mus}

UZ Leuven

Campus Sint Rafaël

Kapucijnenvoer 33

3000 Leuven

Belgium

E-mail:

lisabeth.mus@student.kuleuven.be

ADVERTISEMENT

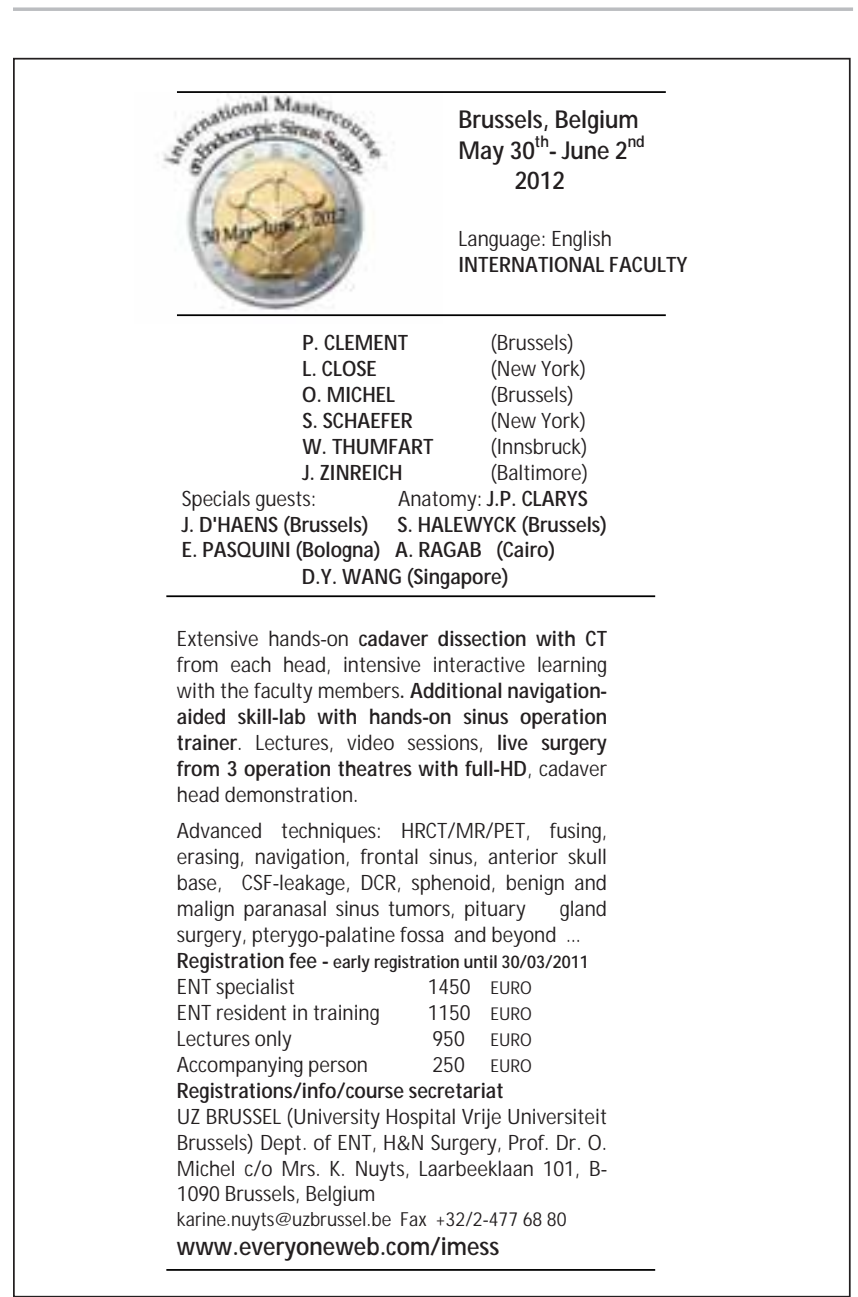

